\title{
Corona fault detection in switchgear with extreme learning machine
}

\author{
Sanuri Ishak ${ }^{1}$, Siaw-Paw Koh ${ }^{2}$, Jian-Ding Tan ${ }^{3}$, Sieh-Kiong Tiong ${ }^{4}$, Chai-Phing Chen ${ }^{5}$ \\ ${ }^{1}$ TNB Research Sdn. Bhd, Malaysia \\ 2,3,4,5Institute of Sustainable Energy, Universiti Tenaga Nasional, Malaysia
}

\section{Article Info \\ Article history: \\ Received Oct 25, 2019 \\ Revised Dec 28, 2019 \\ Accepted Jan 24, 2020}

\section{Keywords:}

Categorization

Corona discharge

Extreme learning machine

Switchgear faults

\begin{abstract}
Switchgear is a very important component in a power distribution line. Failure in switchgear can lead to catastrophic danger and losses. In this research, a fault detection system is proposed with the implementation of Extreme Learning Machine (ELM). This algorithm is capable to identify faults in switchgear by analyzing the sound wave generated. Experiments are carried out to investigate the performance of the developed algorithm in identifying Corona faults in switchgears. The performances are analyzed in time and frequency domains, respectively. In time domain analysis, the results show $90.63 \%, 87.5 \%$, and $87.5 \%$ of success rates in differentiating the Corona and non-Corona cases in training, validation and testing phases respectively. In frequency domain analysis, the results show $89.84 \%, 83.33 \%$, and $87.5 \%$ success rates in training, validation and testing phases respectively. It can thus be concluded that the developed algorithm performed well in identifying Corona faults in switchgears.
\end{abstract}

This is an open access article under the CC BY-SA license.

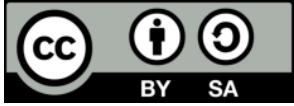

Corresponding Author:

Jian-Ding Tan,

Institute of Sustainable Energy,

Universiti Tenaga Nasional,

Jalan Ikram-Uniten, 43000 Kajang, Selangor, Malaysia.

Email: tjianding @uniten.edu.my

\section{INTRODUCTION}

Switchgear is one of the vital equipment in a power distribution network [1]. In power generating system, switchgears act as a mean to isolate and de-energize specific electrical components and buses to ensure safety of downstream maintenance work, such as faults clearing, routine maintenance, and equipment replacements. They are generally categorized by the insulating medium, such as air or oil, and are typically specified into low, medium, and high voltage classes [2]. It is important to keep a close monitoring on the condition and performance of operating switchgears. Diagnosis and corrective maintenance onto faulty switchgears should be prompt and immediate. A single incident can cause dire effects to the distribution network, operational staffs and thousands of end users, which will in turn cause major spikes on customer interruption statistics and regulatory perception $[3,4]$.

Failures on switchgears are usually caused by gradual degradation of the parts, such as insulators, switches and connectors [5]. At early stage, electrical faults such as corona, surface discharge and arcing can produce noises that are detectable in the frequency range $(20 \mathrm{kHz}$ to $100 \mathrm{kHz})$ of an ultrasonic detection system [6]. Such failures are not easily visible by naked eyes, but it is possible to identify the noise via ultrasonic detection systems [7]. The implementation of ultrasound detection systems can provide utility companies a new approach to ensure improved reliability and performance of critical electrical assets. To date, switchgear faults detection in Malaysia relies heavily on manual random inspection by qualified 
technical experts [8]. An organized soft computing system with growing database can help to ensure a more systematic inspection routine.

Switchgear failures can generally be categorized into several types, such as arcing, tracking, surface discharge and mechanical failure [9]. One of the commonly encountered faults is caused by Corona discharge [10]. Corona is the glow or electrical discharge around conductors [11]. Corona starts almost silently and occurs when the surrounding air is stressed beyond its ionization point without developing flashover [12]. The air between layers of insulation becomes charged when electrical stress exceeds the insulation value of the air. When the humidity and moisture in the air or gas exceeded certain values, Corona discharge occurs to form Ozone and Nitrogen Oxides [13]. These, when combined with the moisture will produce nitric acid, which is destructive to most dielectrics and certain metallic compositions, resulting in corrosion [14]. In addition, the high energy in some discharges result in mechanical, electrical and thermal damage. Corona will only occur when there are over 1000 Volts. It seeks a path to the ground [15]. Left uncorrected, Corona activity can be advancing to the surface discharge stage on the insulation board on a live part. The carbon deposits and light brown discoloration of the insulation board can then be possibly visible by naked eyes by maintenance personnel. Undetected corona can cause further deterioration to the insulator, which in turn leads to other failures such as surface discharge and eventually arcing [16].

This research aims to explore the implementation of soft computing and ultrasonic inspection systems to detect Corona discharge faults at their early stages. This can help the utility companies to take necessary corrective measure to prevent further failures, which can in turn lead to catastrophic losses. In this paper, a modified recognition algorithm enhanced with Extreme Learning Machine (ELM) mechanism is proposed for the detection of corona faults in a switchgear. The major contribution of this research is on the development and implementation of the ELM to identify Corona discharge via the sound waves generated. The layout of the paper is as follows: Chapter two discusses the modified ELM algorithm and its implementation in details. The experimental results and some related discussions are presented in chapter three. The final chapter offers a comprehensive conclusion on the research.

\section{EXTREME LEARNING MACHINE}

Extreme learning machine (ELM) is a competitive machine learning mechanism. It is simple in theory and fast in implementation. Literature study indicates that the ELM has significantly higher learning speed compared to that of a traditional feed-forward network learning algorithms while showing better generalization performance [17-20]. Based on empirical risk minimization theory, the learning process of the ELM requires only a single iteration. Unlike traditional learning algorithms, the ELM shows relatively smaller training error with smaller norm of weight [21]. This, in turn, leads to a better generalization performance [22]. To date, the ELM has shown good performance in regression applications as well as in large dataset classification applications [23-27]. This emerging learning mechanism is gaining popularity due to its robustness, controllability, fast learning rate, and good generalization performance. In this research, the Gaussian Mercer Classifier is incorporated in the ELM as the kernel decision making for the switchgear health condition. The flowchart of the algorithm is as illustrated in Figure 1. The switchgear health condition determination algorithm development in this research can be generally divided into three major phases, namely the training phase, the validation phase, and the prediction of new data phase. Several steps are required to complete each of the phases.

\subsection{Training phase}

The first step of this phase is on the training data collection. Data is collected by using Partial Discharge (PD) Detector. In the concept of ELM, all input data will be restored as shown in (1).

$$
X=\left[\begin{array}{cccc}
X_{11} & X_{12} & \ldots X_{1 M} \\
X_{21} & X_{22} & \ldots & \ldots X_{2 M} \\
\vdots & \vdots & \ddots & \vdots \\
& & & \\
X_{N 1} X_{N 2} & \cdots & & X_{N M}
\end{array}\right]_{N \times M}
$$

In this study, $\mathrm{M}$ is set to 10000 while $\mathrm{N}$ is the number of data sample, yielding (2).

$$
X=\left[\begin{array}{cccc}
X_{11} & X_{12} & \ldots X_{110000} \\
X_{21} & X_{22} & \ldots & X_{210000} \\
\vdots & \vdots & \ddots & \vdots \\
& & & \\
X_{N 1} X_{N 2} & \ldots & & X_{N 10000}
\end{array}\right]_{N \times 10000}
$$




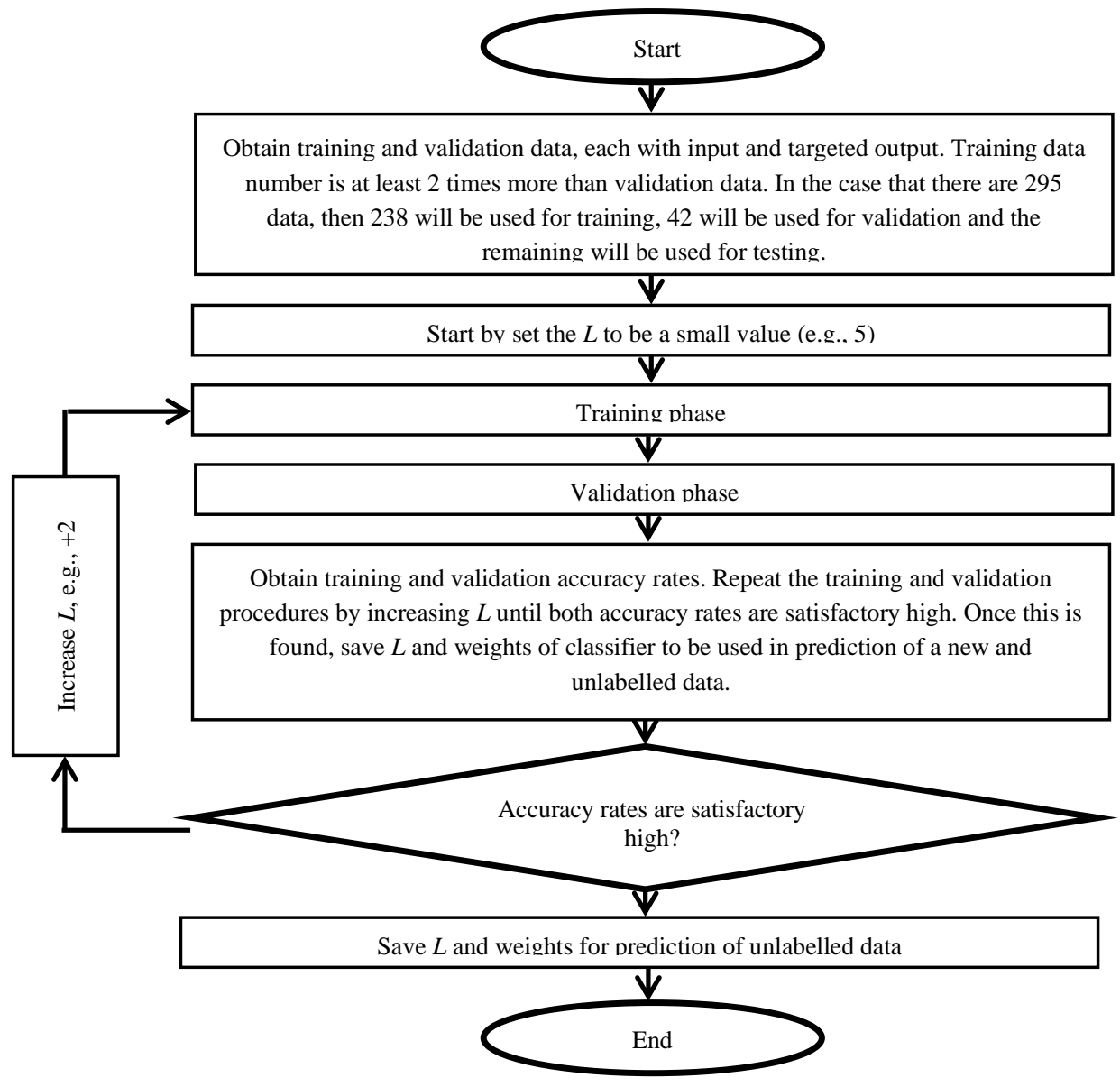

Figure 1. The Flowchart for Main Procedures of ELM Training and Validation

The (3) shows the target output vector, T.

$$
T=\left[\begin{array}{c}
t_{1} \\
t_{2} \\
\vdots \\
t_{N}
\end{array}\right]
$$

The second step is on the initialization. The number of Activation Function, $L$, is defined to be a positive integer value. The type of activation function is defined with the common choice as the Gaussian Radial Basis Function (RBF) or Gaussian Sigmoid function. This is to determine a suitable activation function for the algorithm. An input weight matrix, $a$, is randomly assigned. Upon completion, the activation function is now computed in hidden layer. The Sigmoid function is represented in (4) and (5).

$$
\begin{aligned}
& H_{1}=\frac{1}{1+e^{-\left(a_{i} \cdot x_{j}^{T}+b_{i}\right)}} \\
& H_{1}=\frac{1}{1+e^{-\left(a_{1} \cdot x_{11}+a_{3} \cdot x_{21}+a_{5} \cdot x_{31}+b_{1}\right)}}
\end{aligned}
$$

The same activation formula is used for all nodes. Then, the hidden layer matrix is computed. The matrix and both Sigmoid and RBF functions are defined in (6) and (7) respectively.

$$
\text { Hidden Layer }=\left[\begin{array}{c}
H_{1} \\
H_{2} \\
\vdots \\
H_{N}
\end{array}\right]_{N \times L}
$$




$$
H_{1}=e^{\left\{-b_{i}\left\|x_{j}-a_{i}\right\|^{2}\right\}}
$$

Note that either Sigmoid or RBF function needs to be used. In this project, Sigmoid has been chosen in the programming of the classifier.

In step 4, the output weights are calculated. Under ideal condition, it is assumed that $\mathrm{Y}=\mathrm{Hm}$, where $\mathrm{m}$ is the output weight matrix. The weight can be obtained by $\mathrm{m}=\mathrm{H}^{-1} \mathrm{Y}$. However, the inverse (inv function) matrix above cannot be solved since $\mathrm{H}$ is very likely to be a non-symmetry matrix.To solve this problem, a Moore-Penrose Pseudo inverse matrix (Pinv function) method is employed, yielding (8).

$$
\mathrm{m}=\left(H^{T} H\right)^{-1} \mathrm{H}^{T} \mathrm{Y}
$$

In step 5, the accuracy rate for training data is computed. Once $\beta$ is computed, the same training data is used to calculate the accuracy rate. The output matrix $\mathrm{Y}\left[\begin{array}{llll}y_{1} y_{2} & \ldots & y_{\mathrm{N}}\end{array}{ }^{T}\right.$ was computed based on $\mathrm{H}$, as shown in (9).

$$
\begin{aligned}
& Y \operatorname{signum}(\mathrm{H} \beta) \\
& \operatorname{Signum}(v)=\left\{\begin{array}{cl}
1 & \text { if } v \geq 0 \\
-1 & \text { else }
\end{array}\right.
\end{aligned}
$$

Accuracy rate of training data can be calculated by $100 \% \mathrm{x}$ number of training data that are correctly classified, divided by the number of training data $(N)$. The final step is to save $L$, input and outputs weight $(a$, band $\beta$ ) for validation and prediction phases.

\subsection{Validation phase}

The first step in this phase is to validate the data. A sufficient number of training pairs with $P$ validation samples are collected, each with an input vector and respective target output vector, as shown in (10).

$$
\begin{aligned}
W & =\left[\begin{array}{cccc}
w_{11} & w_{12} & \ldots & w_{1 M} \\
w_{21} & \ldots & \ldots & \ldots \\
\ldots & \ldots & \ldots & \ldots \\
\ldots & \ldots & \ldots & \ldots \\
w_{P 1} & \ldots & \ldots & w_{P M}
\end{array}\right]_{P \times M} \\
D & =\left[\begin{array}{c}
d_{1} \\
d_{2} \\
\ldots \\
d_{P}
\end{array}\right]_{P \times 1}
\end{aligned}
$$

The second step is to load previous information. $L$, input and outputs weight $(a$ band $\beta$ ) are loaded from training phase. In the third step, the hidden layer matrix is calculated. The hidden layer matrix and sigmoid function are defined in (11).

$$
\begin{aligned}
& H_{1}=\frac{1}{1+e^{-\left(a_{i} \cdot x_{j}^{T}+b_{i}\right)}} \\
& \text { Hidden Layer }=\left[\begin{array}{c}
H_{1} \\
H_{2} \\
\vdots \\
H_{P}
\end{array}\right]_{P \times L}
\end{aligned}
$$

Step 4 computes the accuracy rate for validation data. The output matrix $\mathrm{Y}=\left[\begin{array}{lllll}y_{1} & y_{2} & \ldots & y_{P}\end{array}\right]^{\mathrm{T}}$ was computed based on $\mathrm{H}$, while $\mathrm{Y}=\operatorname{signum}(\mathrm{H} \beta)$. The accuracy rate of validation data can be found by multiplying the number of validation data that correctly classified with $100 \%$, divided by the number of validation data $(P)$.

\subsection{Prediction of a new prediction data}

The first step in this phase is to load previous $L$, input and outputs weight $(a$, band $\beta$ ) from the training phase. It is followed by loading new input data, as shown in (12), where $y$ is the classified switchgear health condition based on the input data.

$$
y=\operatorname{signum}(\mathbf{h} \beta)
$$


Information and data from various switchgears were gathered in order to develop the algorithm to identify the corona discharge in the switchgear. Basically, repair information during maintenance and ultrasound data are acquired. The collected ultrasound data were segregated into 314 cases of normal case (no fault) and 228 cases of corona discharge. Noise from ultrasound data were removed before upload it as training data for the ELM model. Figure 2 shows an example of the wave pattern of the sound made during a Corona discharge.

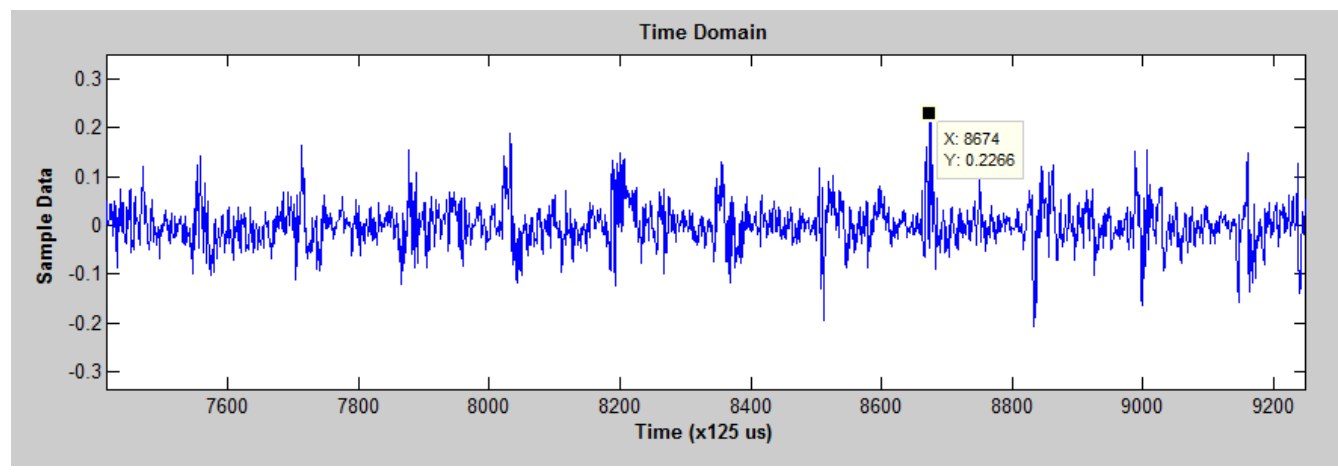

Figure 2. Corona discharge sound wave pattern sample

\section{RESULTS AND ANALYSIS}

Experiments are carried out to test the performance of the developed algorithm in identifying the Corona faults in the switchgears based on the sound waves generated when operating. The experiments are conducted in two different domains, namely in time domain and in frequency domain. The results obtained by the algorithm in training phase, validation phase and testing phase are shown and discussed.

A total of 160 samples of data were used for ELM training, validation and testing in time domain analysis and experiment. The feature number is 10,000 and the hidden neuron number is 1,200. The corona time domain classifier categorizes all data instances of a test dataset as either positive or negative. This classification produces four outcomes - true positive, true negative, false positive and false negative. The classification or corona fault detection accuracy is calculated as the total number of two correct classifications $(\mathrm{TP}+\mathrm{TN})$ divided by the total number of a dataset $(\mathrm{P}+\mathrm{N})$, which is expressed and calculated with the equation as follows:

$$
\begin{array}{rl}
A c c=\frac{T P+T N}{T P+T N+F N+F P} \times 1 & 00 \% \\
& =\frac{T P+T N}{P+N} \times 100 \%
\end{array}
$$

The error rate (ERR) is calculated as the number of all incorrect classifications divided by the total number of the dataset by using the equation as follows:

$$
\begin{array}{rl}
A c c=\frac{F P+F N}{T P+T N+F N+F P} \times 1 & 00 \% \\
& =\frac{F P+F N}{P+N} \times 100 \%
\end{array}
$$

Table 1 shows the output matrix for training phase in time domain. There are 128 sets of data used in the training phase; 26 cases of Corona and 90 cases of non-Corona are successfully identified; 3 cases of Corona are wrongly identified as non-Corona while 9 cases of non-Corona are wrongly identified as Corona. Overall, the accuracy is calculated to be at $90.63 \%$ with the error rate of $9.37 \%$.

In the validation phase of time domain analysis, 24 sets of data are used. Twenty cases of non-Corona are successfully identified. Overall, the accuracy is calculated to be at $87.5 \%$ while the error rate is at $12.5 \%$. Table 2 shows the output matrix. Table 3 shows the output matrix for testing phase in time domain, in which 8 sets of data are used. The algorithm successfully identified 1 Corona fault and all 6 of the non-Corona cases. The testing marks an overall $87.5 \%$ accuracy with $12.5 \%$ error rate. Frequency domain analysis also employed 160 sets of data, in which 128 sets, 24 sets, and 8 sets are used for training, 
validation, and testing phases respectively. Feature number is at 5,000 while hidden neuron number is at 150 for frequency domain analysis. The calculations for the accuracy rates and error rates are the same as in time domain analysis. Table 4 shows the output matrix for training phase in frequency domain. There are 21 cases of Corona and 94 cases of non-Corona successfully identified; 3 cases of Corona are wrongly identified as non-Corona while 10 cases of non-Corona are wrongly identified as Corona. Overall, the accuracy is calculated to be at $89.84 \%$ with the error rate of $10.16 \%$.

In the validation phase of frequency domain analysis, 24 sets of data are used. There are 3 cases of Corona and 17 cases of non-Corona are successfully identified. Overall, the accuracy is calculated to be at $83.33 \%$ while the error rate is at $17.67 \%$. Table 5 shows the output matrix. Table 6 shows the output matrix for testing phase in time domain, in which 8 sets of data are used. The algorithm successfully identified both the Corona faults and 5 non-Corona cases. The testing marks an overall $87.5 \%$ accuracy with $12.5 \%$ error rate.

Table1. Output matrix for training phase: time domain corona fault classification

\begin{tabular}{ccc}
\hline & $\begin{array}{c}\text { Identified to be } \\
\text { Corona }\end{array}$ & $\begin{array}{c}\text { Identified to be } \\
\text { non-Corona }\end{array}$ \\
\hline Actual Corona & 26 & 3 \\
Actual non-Corona & 9 & 90 \\
\hline
\end{tabular}

Table 3. Output matrix for testing phase: time domain corona fault classification

\begin{tabular}{ccc}
\hline & $\begin{array}{c}\text { Identified to be } \\
\text { Corona }\end{array}$ & $\begin{array}{c}\text { Identified to be } \\
\text { non-Corona }\end{array}$ \\
\hline Actual Corona & 1 & 1 \\
Actual non-Corona & 0 & 6 \\
\hline
\end{tabular}

Table 5. Output matrix for validation phase: frequency domain corona fault classification

\begin{tabular}{ccc}
\hline & $\begin{array}{c}\text { Identified to be } \\
\text { Corona }\end{array}$ & $\begin{array}{c}\text { Identified to be } \\
\text { non-Corona }\end{array}$ \\
\hline Actual Corona & 3 & 2 \\
Actual non-Corona & 2 & 17 \\
\hline
\end{tabular}

Table 2. Output matrix for validation phase: time domain corona fault classification

\begin{tabular}{ccc}
\hline & $\begin{array}{c}\text { Identified to be } \\
\text { Corona }\end{array}$ & $\begin{array}{c}\text { Identified to be } \\
\text { non-Corona }\end{array}$ \\
\hline Actual Corona & 1 & 1 \\
Actual non-Corona & 2 & 20 \\
\hline
\end{tabular}

Table 4. Output matrix for training phase: frequency domain corona fault classification

\begin{tabular}{ccc}
\hline & $\begin{array}{c}\text { Identified to be } \\
\text { Corona }\end{array}$ & $\begin{array}{c}\text { Identified to be } \\
\text { non-Corona }\end{array}$ \\
\hline Actual Corona & 21 & 3 \\
Actual non-Corona & 10 & 94 \\
\hline
\end{tabular}

Table 6. Output matrix for testing phase: frequency domain corona fault classification

\begin{tabular}{ccc}
\hline & $\begin{array}{c}\text { Identified to be } \\
\text { Corona }\end{array}$ & $\begin{array}{c}\text { Identified to be } \\
\text { non-Corona }\end{array}$ \\
\hline Actual Corona & 3 & 2 \\
Actual non-Corona & 2 & 17 \\
\hline
\end{tabular}

\section{CONCLUSION}

Switchgear is a component of high importance in a distribution network to ensure safety especially during downstream maintenance. Without proper monitoring and inspection, a switchgear can fail due to many types of faults. A robust fault identification system can be very useful to eliminate manual and random inspections. In this research, a sound-wave-based fault detection system is proposed with the implementation of Extreme Learning Machine (ELM). Experiments are carried out to investigate the performance of the developed algorithm in identifying Corona faults in switchgears. Analysis are carried out in time and frequency domain, respectively. In time domain analysis, the results show 90.63\%, $87.5 \%$, and $87.5 \%$ of success rates in differentiating the Corona and non-Corona cases in training, validation and testing phases respectively. In frequency domain analysis, the results show $89.84 \%, 83.33 \%$, and $87.5 \%$ success rates in training, validation and testing phases respectively. It can thus be concluded that the developed algorithm performed well in identifying Corona faults in switchgears. With the development of the algorithm, the utility companies can have a standard analysis, which in turn grants a more accurate decision making to prioritize the urgency for the remedial works. In time to come, the research can be expended and implemented to identify other switchgear faults, and even other engineering categorization problems.

\section{ACKNOWLEDGEMENTS}

The authors would like to thank Universiti Tenaga Nasional (UNITEN) for the full support of this research under UNIIG and the BOLD initiative. 


\section{REFERENCES}

[1] R. M. A. Velásquez, J. V. M. Lara, and A. Melgar, "Reliability model for switchgear failure analysis applied to ageing," Engineering Failure Analysis, vol. 101, pp. 36-60, 2019.

[2] W. S. Raughley and G. F. Lanik, "Operating experience assessment energetic faults in $4.16 \mathrm{kV}$ to $13.8 \mathrm{kV}$ switchgear and bus ducts that caused fires in nuclear power plants 1986-2001," Tech. Rep., pp. 7-20, 2002.

[3] D. B. Durocher and D. Loucks, "Infrared Windows Applied in Switchgear Assemblies: Taking Another Look," in IEEE Transactions on Industry Applications, vol. 51, no. 6, pp. 4868-4873, Nov.-Dec. 2015.

[4] M. R. B. M. Shariff, M. F. L. Abdullah, M. Y. B. A. Latiff, A. B. Mohamad, and I. B. Ismail, "Detecting leakage current by infrared thermography method," Indonesian Journal of Electrical Engineering and Computer Science, vol. 16, no. 1, pp. 200-207, 2019.

[5] S. Al-Ameri, A. A. Alawady, M. F. M. Yousof, H. Ahmad, Ali. A. Salem, and M. A. Talib, "Frequency response analysis for transformer tap changer damage detection," International Journal of Power Electronics and Drive System, vol. 11, no. 1, 2020.

[6] M. L. Eblen, T. A. Short, and W. J. Lee, "Medium-voltage arc flash in switchgear and live-front transformers," IEEE Trans. Ind. Appl., vol. 52, no. 6, pp. 5280-5288, 2016.

[7] Tan J. D., Dahari M., Koh S. P., Koay Y. Y., and Abed I. A., "Analysis of the Effect of Search Step Size on the Accuracy and Convergence Properties of Electromagnetism-Like Mechanism Algorithm," J. of Mult.-Valued Logic \& Soft Computing,vol. 28, pp. 429-441, 2017.

[8] M. R. B. M. Shariff, M. F. L. Abdullah, M. Y. B. A. Latiff, G. B. M. Tion, Z. A. Haron, and A. Johari, "Impact of EMI Filter installed in AC UPS System to Earth Leakage Current," in 2014 IEEE 8th International Power Engineering and Optimization Conference (PEOCO2014), 2014, pp. 647-652.

[9] A. Krupa, J. Podliński, J. Mizeraczyk, and A. Jaworek, "Velocity field of EHD flow during back corona discharge in electrostatic precipitator," Powder Technology, vol. 344, pp. 475-486, 2019.

[10] P. Cooperman, "Back corona and relaxation time," IEEE Trans. Ind. Appl., vol. 12, no. 1, pp. 84-85, 1976.

[11] J. A. Cross, "An analysis of the current in a point-to-plane corona discharge and the effect of a back-ionizing layer on the plane," J. Phys. D: Apply. Phys., vol. 18, pp. 2463-2471, 1976.

[12] A. Berendt and J. Mizeraczyk, "Transition of the electrohydrodynamic two-phase flow into the single-phase flow in a needle-to-plate negative corona discharge in the finite-volume chamber," J. Electrost., vol. 84 pp. 90-96, 2016.

[13] J. A. Cross, "Back ionization in a negative point-to-plane corona discharge," J. Electrost., vol. 18, pp. 327-344, 1986.

[14] T. Czapka and R. Kacprzyk, "Non-thermal plasma reactor with back corona discharge electrode," J. Phys. Conf. Ser. pp. 301, 2011.

[15] T. Czech, A. T. Sobczyk, A. Jaworek, and A. Krupa, "Corona and back discharges in flue-gas simulating mixture," J. Electrost.,vol. 70, pp. 269-284, 2012.

[16] J. Mizeraczyk, A. Berendt, and J. Podliński, "Temporal and spatial evolution of EHD particle flow onset in air in a needle-to-plate negative DC corona discharge," J. Phys. D. Appl. Phys., vol. 49, 2016.

[17] I. Bahiuddin, A. Y. A. Fatah, S. A Mazlan, M. I. Shapiai, F. Imaduddin, Ubaidillah, D. Utami, and M. N. Muhtazaruddin, "Comparing the linear and logarithm normalized extreme learning machine in flow curve modeling of magnetorheological fluid," Indonesian Journal of Electrical Engineering and Computer Science., vol. 13, no. 3, pp. 1065-1072, 2019.

[18] N. Alias, C. F. M. Foozy, and S. N. Ramli, "Video spam comment features selection using machine learning techniques," Indonesian Journal of Electrical Engineering and Computer Science., vol. 15, no. 2, pp. 1046-1053, 2019.

[19] Y. Cai, X. Liu, Y. Zhang, and Z. Cai, "Hierarchical ensemble of Extreme Learning Machine," Pattern Recognition Letters, vol. 116, pp. 101-106, 2018.

[20] L. Imen and L. Djamel, "Power flow variation based on extreme learning machine algorithm in power system," International Journal of Power Electronics and Drive System, vol. 10, no. 3, pp. 1244-1254, 2019.

[21] T. Jing, A. Maklakov, A. Radionov, S. Baskov, and A. Kulmukhametova, "Research on hybrid SHEPWM based on different switching patterns," International Journal of Power Electronics and Drive System, vol. 10, no. 4, pp. 1875-1884, 2019.

[22] Y. Zhang, J. Wu, Z. Cai, P. Zhang, and L. Chen, "Memetic extreme learning machine," Pattern Recognit., vol. 58, pp. 135-148, 2016.

[23] J. Tang, C. Deng, and G. -B. Huang, "Extreme learning machine for multilayer perceptron," IEEE Trans. Neural Netw. Learn. Syst, vol. 27, pp. 809-821, 2016.

[24] R. Minhas, A. Baradarani, S. Seifzadeh, and Q. M. J. Wu, "Human action recognition using extreme learning machine based on visual vocabularies," Neurocomputing, vol. 73, pp. 1906-1917, 2010.

[25] W. Zong and G.-B. Huang, "Face recognition based on extreme learning machine," Neurocomputing, vol. 74, pp. 2541-2551, 2011.

[26] Y. Zeng, X. Xu , D. Shen, Y. Fang, and Z. Xiao, "Traffic sign recognition using kernel extreme learning machines with deep perceptual features," IEEE Trans. Intell. Transp. Syst., vol. 18, pp. 1647-1653, 2017.

[27] Y. Yang and Q. M. J. Wu, "Multilayer extreme learning machine with subnet-work nodes for representation learning," IEEE Trans. Cybern, vol. 46, pp. 2570-2583, 2016. 